\title{
Direct Separation of Short Range Order in Intermixed Nanocrystalline and Amorphous Phases
}

\author{
Anatoly I. Frenkel* \\ Physics Department, Yeshiva University, New York, New York 10016 \\ Alexander V. Kolobov \\ Joint Research Center for Atom Technology and Laboratory for Advanced Optical Technology, \\ National Institute for Advanced Industrial Science and Technology, 1-1-1 Higashi, Tsukuba, Ibaraki 305-8562, Japan \\ Ian K. Robinson \\ Loomis Laboratory, Physics Department, University of Illinois at Urbana-Champaign, Urbana, Illinois 61801 \\ Julie O. Cross \\ PNC-CAT, Argonne National Laboratory, 9700 South Cass Avenue, Argonne, Illinois 60439 \\ Yoshihito Maeda \\ Osaka Prefecture University, Osaka 599-8531, Japan \\ Charles E. Bouldin \\ Ceramics Division, National Institute of Standards, Gaithersburg, Maryland 20899
}

(Received 29 May 2002; published 27 December 2002)

\begin{abstract}
Diffraction anomalous fine-structure (DAFS) and extended x-ray absorption fine-structure (EXAFS) measurements were combined to determine short range order (SRO) about a single atomic type in a sample of mixed amorphous and nanocrystalline phases of germanium. EXAFS yields information about the SRO of all Ge atoms in the sample, while DAFS determines the SRO of only the ordered fraction. We determine that the first-shell distance distribution is bimodal; the nanocrystalline distance is the same as the bulk crystal, to within 0.01(2) $\AA$, but the mean amorphous Ge-Ge bond length is expanded by $0.076(19) \AA$. This approach can be applied to many systems of mixed amorphous and nanocrystalline phases.

DOI: $10.1103 /$ PhysRevLett.89.285503

PACS numbers: 81.07.Bc, 61.10.Ht, 61.43.Dq, 61.46.+w
\end{abstract}

Materials consisting of a mixture of crystalline and amorphous phases and crystallization of amorphous materials and glasses are very important subjects in modern physics and technology. An example of the former is nanocrystalline silicon whose importance in Si technology continuously grows. Since transport of carriers is determined by the amorphous phase surrounding the nanocrystals, the knowledge of its structure is of utmost importance both for investigation of the transport behavior in heterogeneous systems and for applications. Examples of importance of the crystallization are commercial CDs and DVDs which are based on amorphousto-crystalline phase transitions in the Ge-Sb-Te system. In order to increase the crystallization and amorphization rates for faster and denser optical recording, knowledge of the bond rearrangement on the atomic scale is vital. While generally the short range order of glasses is the same as that of the corresponding crystals, exceptions do exist. An example is GeTe which is an important constituent of the commercially used media. While its crystal structure is definitely known and possesses $3(\mathrm{Ge}): 3(\mathrm{Te})$ coordination, the amorphous phase has been a matter of controversy for more than two decades with both
$3(\mathrm{Ge}): 3(\mathrm{Te})$ coordination and $4(\mathrm{Ge}): 2(\mathrm{Te})$ coordination being supported by various techniques [1]. The obvious importance of this material for optical memories and optical data storage requires a detailed investigation of the crystallization/amorphization processes.

Annealing of amorphous semiconductors often leads to formation of nanocrystallites embedded in an amorphous matrix. The relative amount and average size of crystallites, as well as many physical properties changed by annealing [2], depend on elemental composition ( $\mathrm{Ge}, \mathrm{Si}$, $\mathrm{Ge}-\mathrm{Si}$ alloys, $\mathrm{As}_{2} \mathrm{~S}_{3}, \mathrm{As}_{2} \mathrm{Se}_{3}, \mathrm{GeSe}_{2}$, etc.) and the details of sample preparation. However, quantitative information about the structures of both the crystalline and amorphous states is incomplete because previously used structural techniques did not possess sensitivities to both the short range (SRO) and the long range order (LRO) correlations of the same atomic type in a multiphase mixture.

Separating the structure of the coexisting amorphous (a) and nanocrystalline (nc) phases has been attempted previously using x-ray diffraction (XRD) [3], Raman scattering [3], and extended $x$-ray absorption fine structure (EXAFS) [4], but detailed knowledge about the structure of each phase, i.e., the number of nearest 
neighbors, interatomic distances, and local geometry, could not be obtained. One attempt to discriminate the structural contributions of the same element $(\mathrm{Ge})$ in a mixture of its crystalline and amorphous phases used EXAFS photoelectron wave number $k$-space selectivity, where only the nc phase has higher shell EXAFS above $k \sim 3 \AA^{-1}[5,6]$. However, this missed important information such as a separate determination of the a Ge and nc Ge first-neighbor distances, since both a and nc phases give first-neighbor contributions to the SRO, and only the average distance over both types of Ge-Ge correlations can then be determined [7].

In this work, we demonstrate that a relatively new structural technique, diffraction anomalous fine structure (DAFS) [8], the hybrid of the $\mathrm{x}$-ray diffraction and $\mathrm{x}$-ray absorption measurements that combines the LRO sensitivity of the former and the SRO sensitivity of the latter, can be used to disentangle the contributions of ordered and disordered states of the same element. This extends the use of the EXAFS $k$ space selectivity to include variable photon momentum transfer, $q$, in a manner that is complementary to differential anomalous scattering (DAS). DAS fixes photon energy $E$ at a few values and scans $q$ as a continuous variable in order to achieve a chemical separation of the SRO, e.g., around different atomic types in a single-phased amorphous material [9]. Here, to separate the SRO of a single atomic type in a mixture of its ordered and disordered phases, we interchange the variables, fixing $q$ at a few values (note that EXAFS has the information content of $q=0$ DAFS) and scanning $E$ as a continuous variable.

Using XRD, one can verify the presence of nanocrystallites, and obtain their average size and lattice parameter. In the DAFS experiment, a diffraction peak specific to the ordered nc regions is measured as a function of incident $\mathrm{x}$-ray energy in the anomalous scattering region, across the Ge absorption edge. The resultant oscillatory fine-structure signal $\chi_{\mathrm{nc}}(k)$ is due to local structural correlations in only the ordered nc regions. Independently measured EXAFS $\chi_{\text {total }}(k)$ of the same sample yields SRO averaged over all Ge atoms, therefore the SRO from $\mathrm{nc} \mathrm{Ge}$ and from all other $n-1$ structurally inequivalent Ge environments in the sample can be separated by appropriate combinations of $\chi_{\text {total }}(k)$ and $\chi_{\mathrm{nc}}(k)$ :

$$
\chi_{\text {total }}(k)=\alpha_{1} \chi_{\mathrm{nc}}(k)+\sum_{i=2}^{n} \alpha_{i} \chi_{\text {other }}^{i}(k),
$$

where $\chi_{\text {other }}^{i}(k)$ is partial EXAFS due to the $i$ th state of Ge other than nanocrystalline (e.g., $\mathrm{Ge}-\mathrm{O}$ and a $\mathrm{Ge}$ ) and $\alpha_{i}$ are weighting coefficients: $\sum_{i=1}^{n} \alpha_{i}=1$.

We studied the local structure of mixed phase samples containing amorphous and nanocrystalline germanium supported by an $\mathrm{SiO}_{2}$ matrix. Two samples were prepared by codeposition of $\mathrm{Ge}$ and $\mathrm{Si}$ oxides onto quartz substrates by radio-frequency magnetron sputtering [10]. The Ge concentration was $60 \mathrm{~mol} \%$ and the thickness of the samples was $500 \mathrm{~nm}$. One of the samples was then annealed for $1 \mathrm{~h}$ at $800^{\circ} \mathrm{C}$ in an argon atmosphere. TEM studies of these samples have demonstrated that nanocrystals of ca. 15-20 nm in size, roughly spherical in shape [Fig. 1(a)], were formed in the annealed sample [11]. Combining these results with those of Raman scattering [12] and EXAFS [11] experiments performed with the same samples, we concluded that, in the Ge-rich phase embedded in the $\mathrm{SiO}_{2}$ matrix of both the asprepared and annealed samples, Ge-Ge and Ge-O correlations dominate and $\mathrm{Ge}-\mathrm{Si}$ alloying is negligible. However, there are three different structural environments around the Ge in the annealed sample which complicate the analysis: Ge nanocrystals, amorphous $\mathrm{Ge}$, and Ge oxide. Moreover, the coexistence of amorphous and crystalline phases of $\mathrm{Ge}$ squeezed into the small regions (several tens of $\mathrm{nm}$ ) in the $\mathrm{SiO}_{2}$ host matrix should result in a large surface/volume ratio of Ge-Ge bonds in a $\mathrm{Ge}$ near the interface with the nc Ge phase. As modeled previously for $\mathrm{Si}$, these amorphous interface layers may be $\sim 7 \AA$ thick [13]. Therefore, the average Ge-Ge bond length in the amorphous $G e$ phase in our sample is expected to differ from the average Ge-Ge bond lengths in either bulk crystalline or amorphous Ge phases. In order to isolate all three contributions, we analyzed the samples by three different $\mathrm{x}$-ray techniques, as described below.

All three experiments, XRD, DAFS, and EXAFS, were performed at room temperature using a custom-designed four-circle Kappa diffractometer at Brookhaven National Laboratory's National Synchrotron Light Source on Beamline X16C. Diffraction measurements were taken first, to determine the relative fractions of amorphous and crystalline phases in the as-deposited and annealed
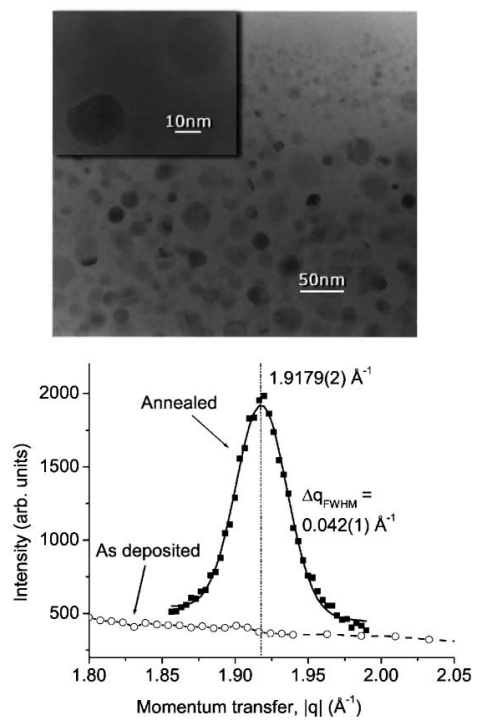

FIG. 1. Determination of the average size and shape of the crystallites: (a) TEM image of an annealed sample: Ge nanocrystallites embedded in $\mathrm{SiO}_{2}$. (b) XRD measurements of the Ge(111) peak. The same peak was used later for DAFS measurements. 
samples. The as-deposited sample was verified as amorphous [Fig. 1(b)]. The Bragg peak profiles of the annealed sample were measured by the $\theta-2 \theta$ method at the (111), (220), and (311) peaks, which had nearly identical $\Delta q_{\mathrm{FWHM}}$ values of $0.042(1) \AA^{-1}$, indicating that the peak broadening is due to the finite-size effect of roughly spherical ordered regions. No preferred orientation of the crystallites was observed, in agreement with our TEM studies [Fig. 1(a)]. The characteristic length $L$ of the ordered regions was estimated from the measured $\Delta q_{\mathrm{FWHM}}$ using the Scherrer formula $(L=0.94 \times 2 \pi /$ $\left.\Delta q_{\text {FWHM }}\right)$ as ca. $14 \pm 1 \mathrm{~nm}$, in agreement with the TEM results above.

For DAFS and EXAFS measurements, energy scans of the (111) reflection (Fig. 2) were performed from $200 \mathrm{eV}$ below to $600 \mathrm{eV}$ above the $\mathrm{Ge} K$ edge energy (11 $104 \mathrm{eV})$. The $\mathrm{x}$-ray beam was focused at the sample position using a sagittally focused second crystal of the $\mathrm{Si}(111)$ monochromator. In the DAFS experiment, the energy dependence of the Bragg peak intensity was measured in each of 20 consecutive scans. The EXAFS measurements, also used to isolate the DAFS signal from the fluorescence background, were taken in the same scattering geometry as the DAFS experiment, by moving the $2 \theta$ motor of the diffractometer off of the Bragg peak and measuring the Ge $K_{\alpha}$ fluorescence intensity as a function of incident $\mathrm{x}$-ray energy. To calibrate $\mathrm{x}$-ray energy between consecutive scans, we monitored the absorption of air-scattered radiation in a reference Ge filter [14].

After isolating the $f^{\prime}(E)$ and $f^{\prime \prime}(E)$ from the Bragg intensity (Fig. 2, insets) using the iterative KramersKronig algorithm [15], the total absorption cross section $\sigma(E)$ was obtained using the optical theorem: $\sigma=$ $4 \pi \hbar c f^{\prime \prime}(E) / E$. In the EXAFS experiment, this quantity was obtained directly, from the ratio of the fluorescent and incident beam intensities. The appearance of the shoulder in the near-edge region of $f^{\prime \prime}(E)$ (arrow in Fig. 2) is a known signature of crystalline Ge. We find

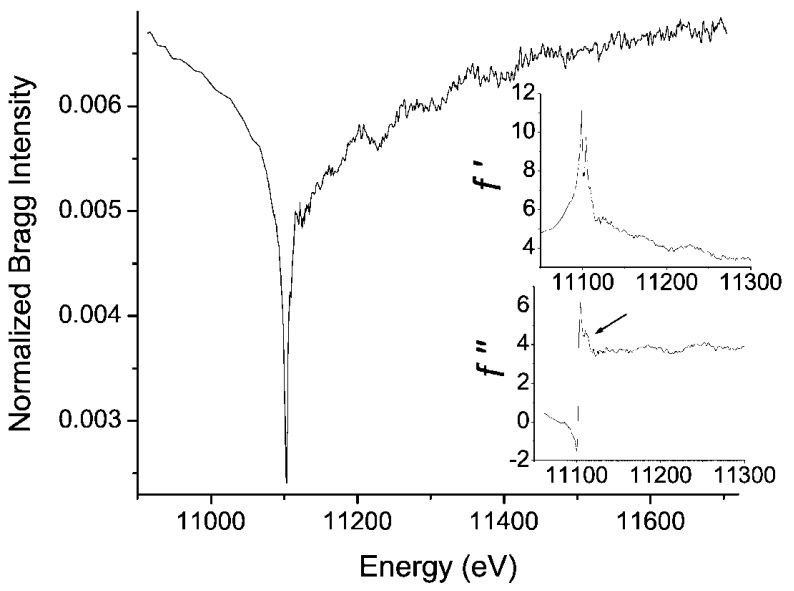

FIG. 2. Raw DAFS and $f^{\prime}, f^{\prime \prime}$ data (insets) separated by the Kramers-Kronig transforms. A near-edge "shoulder" in the $f^{\prime \prime}$ data is indicated by an arrow. that we can qualitatively reproduce, using ab initio FEFF8 [16] simulations, the main near-edge features, including the "shoulder," for clusters of atoms larger than $15 \AA$ in diameter. This confirms that the $\mathrm{nc}$ Ge is ordered on at least this length scale, in agreement with the XRD and TEM results. After the background subtraction and edgestep normalization of two sets of raw $\sigma(E)$ data measured separately by EXAFS and DAFS, their respective $k \chi(k)$ signals were obtained (Fig. 3). The clearest difference between the curves is the absence of the Ge-O coordination in the DAFS-extracted $k \chi(k)$ data, evident in their Fourier transforms (Fig. 4), illustrating the phase selectivity of DAFS technique. Fitting EXAFS theory calculated using FEFF7 [17] software to the fluorescence EXAFS data (Fig. 4) yields a mean Ge-Ge distance, averaged over both a and nc Ge, of 2.476(7) A.

From the analysis of the DAFS-extracted nc-Ge $k \chi(k)$ data (Fig. 4), we obtained the nc Ge-Ge distances to be 2.44(2) $\AA$, in agreement with those measured directly by $\mathrm{XRD}[2.455(1) \AA]$. The nc Ge-Ge distance is shorter than that of the EXAFS-extracted average Ge-Ge distance, indicating that the average Ge-Ge distance distribution is bimodal, shorter in the crystalline, and longer in the rest of the Ge-rich phase. This can be visualized by comparing the residuals obtained after fitting the GeGe contribution to the bulk EXAFS data with the unimodal (UNI) and bimodal (BI) distribution models (Fig. 4). As Fig. 4 illustrates, the fit residuals obtained for the UNI model are twice greater than in the BI model. Moreover, the misfit of the UNI model in the vicinity of Ge-Ge peak is concentrated on the right-hand side of the bulk peak, i.e., manifesting the longer amorphous $\mathrm{Ge}-\mathrm{Ge}$ pair length, unaccounted for in the UNI model.

Analysis of the signal of the nc Ge phase (obtained from the DAFS data) and the cumulative signal containing a mixture of $\mathrm{GeO}$, a $\mathrm{Ge}$, and nc $\mathrm{Ge}$ (measured by fluorescence EXAFS) allows separation of the structural contributions of all three phases. We used constrained

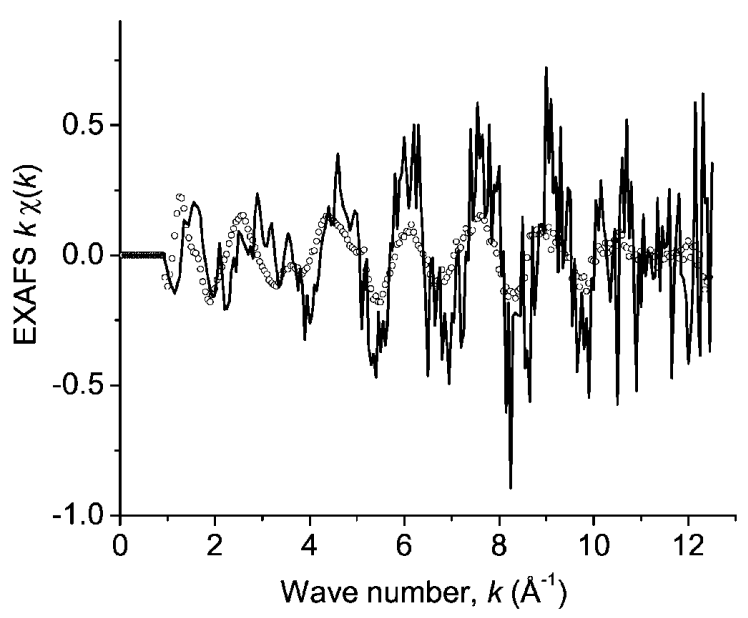

FIG. 3. $k$-weighted EXAFS measured by bulk fluorescence (symbols) and extracted from the DAFS $f^{\prime \prime}$ (solid line).

285503-3 


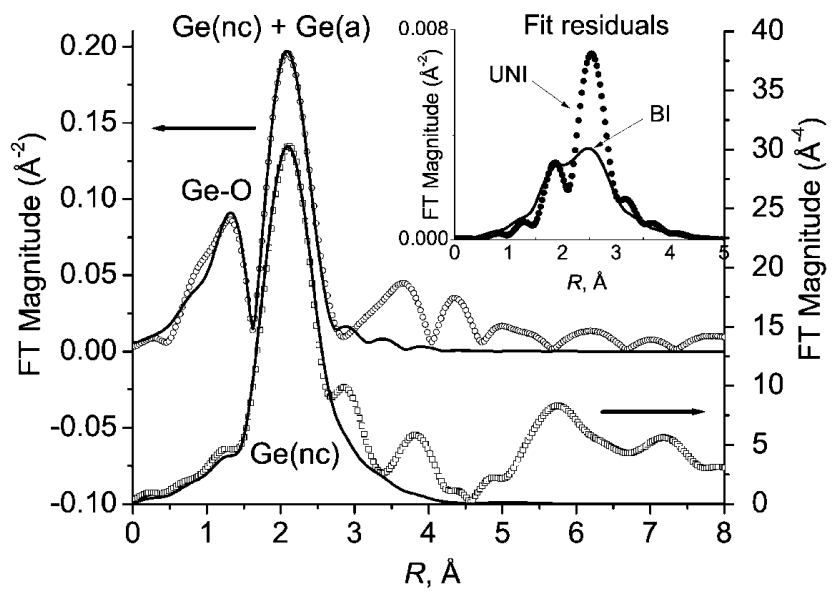

FIG. 4. Fourier transform (FT) magnitudes of the FEFF7 theory (solid) and the data (symbols). Fits were performed in $r$ space using the $k$ range from 3.5 to $10 \AA^{-1}$. Top left: Bulk data and theory for Ge-O and average Ge-Ge contributions; $k$ weighting was used in FT. Bottom left: DAFS-extracted data and theory for the nc Ge-Ge only; $k^{3}$ weighting was used in FT. The signal above ca. $3 \AA$ in the bottom plot is dominated by noise in the $\chi_{\mathrm{nc}}(k)$ data (Fig. 3) preventing the analysis of higher shells characteristic for the crystalline structure $[5,6]$. The inset shows the FT magnitudes of the fit residuals in the vicinity of the Ge peak obtained after fitting bulk EXAFS data (top left) with the unimodal Ge-Ge distribution model (UNI) and the bimodal distribution model (BI).

fitting of the total EXAFS with a model that included all three contributions: Ge-O, nc Ge-Ge, and a Ge-Ge, but with all nc-Ge structural parameters fixed at values previously determined from the DAFS. From this, the fractions of all three components were obtained: Ge-O, 23(2)\%; nc Ge-Ge, 43(18)\%; a Ge-Ge, 34(18)\%. In addition to the previously obtained first-neighbor nc Ge-Ge distance of 2.44(2) $\AA$, the remaining two distances were determined as 1.746(5) $\AA$ in Ge-O, and 2.516(18) $\AA$ in a Ge. The obtained Ge-O distance is in good agreement with that reported previously for a similarly prepared sample [11]. The mean Ge-Ge distance in a Ge is within the 2.47-2.54 A range quoted in previous works on amorphous germanium [18]. The reason this distance is $0.076(19) \AA$ A longer than that in the $\mathrm{nc} \mathrm{Ge}$ is believed to be caused by the large contribution of $\mathrm{Ge}-\mathrm{Ge}$ bonds located within the distorted amorphous-crystalline interface layers, as described above.

This work illustrates that a combination of DAFS and EXAFS can be used to determine the SRO around a single atomic type in a sample of mixed amorphous and nanocrystalline phases. DAFS determines the SRO of only the ordered phase, while EXAFS measures the SRO of both phases; the combination of both tools allows a full separation of the SRO. In this Letter, we demonstrate this separation in a monatomic system of mixed a and nc $\mathrm{Ge}$, but the approach can be generalized to make separate SRO determinations in multiatomic systems containing mixed amorphous and crystalline phases. Our approach relies on no specific characteristics of the sample except the presence of an accessible $\mathrm{x}$-ray absorption edge for both absorption and diffraction experiments. Possible applications include studies of materials such as quantum dots, ion-damaged materials, and thin-film oxides, where the nucleation and growth of nanocrystals from an amorphous matrix are important.

The authors acknowledge the support of NSLS by the Divisions of Materials and Chemical Sciences of DOE and support of Beamline X16C by the Seitz Materials Research Laboratory, University of Illinois at UrbanaChampaign (US-DOE Contract No. DEFG02-91ER45439).

*Author to whom correspondence should be addressed. Electronic mail: afrenke2@ymail.yu.edu

[1] S. Hosokawa et al., J. Phys. Condens. Matter 10, 1931 (1998).

[2] M. A. Paesler, S.C. Agarwal, S. J. Hudgens, and H. Fritzsche, in Tetrahedrally Bonded Amorphous Semiconductors, edited by M.H. Brodsky, S. Kirkpatrick, and D. Weaire, AIP Conf. Proc. No. 20 (AIP, New York, 1974), p. 37.

[3] F. Evangelisti, M. Garozzo, and G. Conte, J. Appl. Phys. 53, 7390 (1982).

[4] R. J. Nemanich, G. A. N. Connell, T. M. Hayes, and R. A. Street, Phys. Rev. B 18, 6900 (1978).

[5] E. A. Stern, C. E. Bouldin, B. von Roedern, and J. Azoulay, Phys. Rev. B 27, 6557 (1983).

[6] C. E. Bouldin, E. A. Stern, B. von Roedern, and J. Azoulay, Phys. Rev. B 30, 4462 (1984).

[7] The possibility to separately measure, with EXAFS, the a-Ge and nc-Ge bond lengths exists, theoretically, if their split $\Delta R$ is sufficiently large for the beat in $k$ space of EXAFS data, occurring at $\pi /(2 \Delta R)$ to be detected. If $\Delta R$ is of the order of $0.05 \AA$ as in the case here, the beat must occur at $30 \AA^{-1}$, which is far beyond reach in the XAFS experiment.

[8] H. Stragier et al., Phys. Rev. Lett. 69, 3064 (1992); I. J. Pickering, M. Sansone, J. Marsch, and G. N. George, J. Am. Chem. Soc. 115, 6302 (1993).

[9] P. H. Fuoss, P. Eisenberger, W. K. Warburton, and A. Bienenstock, Phys. Rev. Lett. 46, 1537 (1981).

[10] Y. Maeda, Phys. Rev. B 51, 1658 (1995).

[11] A.V. Kolobov, H. Oyanagi, Y. Maeda, and K. Tanaka, J. Synchrotron Radiat. 8, 511 (2001).

[12] A. V. Kolobov, Y. Maeda, and K. Tanaka, J. Appl. Phys. 88, 3285 (2000).

[13] N. Bernstein, M. J. Aziz, and E. Kaxiras, Phys. Rev. B 58, 4579 (1998).

[14] J. O. Cross and A. I. Frenkel, Rev. Sci. Instrum. 70, 38 (1999).

[15] J. O. Cross, W. T. Elam, J. C. Woicik, and L. B. Sorensen, J. Synchrotron Radiat. 6, 335 (1999).

[16] A. L. Ankudinov, B. Ravel, J. J. Rehr, and S. D. Conradson, Phys. Rev. B 58, 7565 (1998).

[17] S. I. Zabinsky et al., Phys. Rev. B 52, 2995 (1995).

[18] Y. Waseda, The Structure of Non-Crystalline Materials (McGraw-Hill, New York, 1980). 